\title{
Algebraic Independence over Positive Characteristic: New Criterion and Applications to Locally Low Algebraic Rank Circuits
}

\author{
Anurag Pandey ${ }^{1}$, Nitin Saxena $^{2}$, and Amit Sinhababu ${ }^{3}$
}

1 MPI for Informatics \& Saarland University, Department of Computer Science, Saarbrücken, Germany

apandey@mpi-inf .mpg.de

2 Department of CSE, Indian Institute of Technology Kanpur, Kanpur, India

nitin@cse.iitk.ac.in

3 Department of CSE, Indian Institute of Technology Kanpur, Kanpur, India

amitks@cse.iitk.ac.in

\begin{abstract}
The motivation for this work comes from two problems- test algebraic independence of arithmetic circuits over a field of small characteristic, and generalize the structural property of algebraic dependence used by (Kumar, Saraf CCC'16) to arbitrary fields.

It is known that in the case of zero, or large characteristic, using a classical criterion based on the Jacobian, we get a randomized poly-time algorithm to test algebraic independence. Over small characteristic, the Jacobian criterion fails and there is no subexponential time algorithm known. This problem could well be conjectured to be in RP, but the current best algorithm puts it in NP\#P (Mittmann, Saxena, Scheiblechner Trans.AMS'14). Currently, even the case of two bivariate circuits over $\mathbb{F}_{2}$ is open. We come up with a natural generalization of Jacobian criterion, that works over all characteristic. The new criterion is efficient if the underlying inseparable degree is promised to be a constant. This is a modest step towards the open question of fast independence testing, over finite fields, posed in (Dvir, Gabizon, Wigderson FOCS'07).

In a set of linearly dependent polynomials, any polynomial can be written as a linear combination of the polynomials forming a basis. The analogous property for algebraic dependence is false, but a property approximately in that spirit is named as "functional dependence" in (Kumar, Saraf CCC'16) and proved for zero or large characteristic. We show that functional dependence holds for arbitrary fields, thereby answering the open questions in (Kumar, Saraf CCC'16). Following them we use the functional dependence lemma to prove the first exponential lower bound for locally low algebraic rank circuits for arbitrary fields (a model that strongly generalizes homogeneous depth-4 circuits). We also recover their quasipoly-time hitting-set for such models, for fields of characteristic smaller than the ones known before.

Our results show that approximate functional dependence is indeed a more fundamental concept than the Jacobian as it is field independent. We achieve the former by first picking a "good" transcendence basis, then translating the circuits by new variables, and finally approximating them by truncating higher degree monomials. We give a tight analysis of the "degree" of approximation needed in the criterion. To get the locally low algebraic rank circuit applications we follow the known shifted partial derivative based methods.
\end{abstract}

1998 ACM Subject Classification I.1 Symbolic and Algebraic Manipulation, F.2 Analysis of Algorithms and Problem Complexity

Keywords and phrases independence, transcendence, finite field, Hasse-Schmidt, Jacobian, differential, inseparable, circuit, identity testing, lower bound, depth-4, shifted partials

Digital Object Identifier 10.4230/LIPIcs.MFCS.2016.74

ㅇ) Anurag Pandey, Nitin Saxena, and Amit Sinhababu;
licensed under Creative Commons License CC-BY

41st International Symposium on Mathematical Foundations of Computer Science (MFCS 2016). Editors: Piotr Faliszewski, Anca Muscholl, and Rolf Niedermeier; Article No. 74; pp. 74:1-74:15

Leibniz International Proceedings in Informatics

LIPICS Schloss Dagstuhl - Leibniz-Zentrum für Informatik, Dagstuhl Publishing, Germany 


\section{Introduction}

Algebraic dependence is a fundamental concept in algebra that captures algebraic/polynomial relationship of objects like numbers, polynomials, rational functions or power series, over some field. Here we define algebraic dependence of polynomials, since in this work we deal only with polynomials. Polynomials $f_{1}, \ldots, f_{m} \in \mathbb{F}\left[x_{1}, \ldots, x_{n}\right]$ are called algebraically dependent over field $k$ if and only if there exists a nonzero polynomial $A\left(y_{1}, \ldots, y_{m}\right) \in \mathbb{F}\left[y_{1}, \ldots, y_{m}\right]$ such that $A\left(f_{1}, \ldots, f_{m}\right)=0$ and such an $A$ is called an annihilating polynomial of $f_{1}, \ldots, f_{m}$. If no such nonzero polynomial $A$ exists, the given polynomials are called algebraically independent over $k$.

For example, $f_{1}=(x+y)^{2}$ and $f_{2}=(x+y)^{3}$ are algebraically dependent over any field, as $y_{1}^{3}-y_{2}^{2}$ is an annihilating polynomial. Polynomials $x+y$ and $x^{p}+y^{p}$ are dependent over $\mathbb{F}_{p}$, but independent over $\mathbb{Q}$. Monomials $x_{1}, \ldots, x_{n}$ are examples of algebraically independent polynomials over any field.

Algebraic dependence can be viewed as a generalization of linear dependence as the former captures algebraic relationships of any degree, whereas the latter captures linear relationships. Algebraic dependence shares a few combinatorial properties (known as matroid properties [30]) with linear dependence. For example, if a set of polynomials are algebraically independent then any subset of them are algebraically independent. The transcendence degree (trdeg or algRank) of a set of polynomials is defined as the maximal number of algebraically independent polynomials and it is well defined thanks to the matroid properties. The concepts of rank and basis in linear algebra have analogs here as transcendence degree and transcendence basis respectively.

The concept of algebraic independence is useful in several areas of mathematics: field theory, commutative algebra, algebraic geometry, invariant theory, theory of algebraic matroids. It has found interesting applications in computer science as well. For example, [28] used algebraic dependence in analysis of program invariants of arithmetic straight line programs. To prove lower bounds on the formula size of determinant, [21] also used transcendence degree as a tool. [7, 9] constructed explicit deterministic randomness extractors for sources which are polynomial maps over finite fields. [8] gives a cryptography application, using algebraic characterization of entropy of low degree polynomials. [4, 1, 27] used it for designing faster deterministic hitting-sets for some interesting cases of the polynomial identity testing problem (PIT) and proving circuit lower bounds. [5] used algebraic independence of polynomials to show the hardness of a parameterized counting problem.

An example relevant to computer science is to compute the "entropy" of a given polynomial map $\phi:\left(x_{1}, \ldots, x_{n}\right) \mapsto\left(f_{1}, \ldots, f_{n}\right)$ in the space $\mathbb{F}_{q}^{n}$, where $q$ is a power of $p=2$ (more, generally, $p$ grows as a polynomial in the input size). This turns out to be a question of computing the transcendence degree of the polynomials $f_{1}, \ldots, f_{n}$ [7]. For constant $p$, there are no good methods known. Our work improves the state of the art in this regime.

To discuss the complexity of algebraic independence testing, we have to specify the representation of input polynomials. An arithmetic circuit is a directed acyclic graph consisting of addition $(+)$ and multiplication $(\times)$ gates as nodes, takes variables $x_{1}, \ldots, x_{n}$ and field constants as input (leaves), and outputs a polynomial $f\left(x_{1}, \ldots, x_{n}\right)$. This is a succinct representation of multivariate polynomials, as polynomials of high degree (or having many monomials) can be represented by small circuits.

Perron [31, 32] gave a bound on the degree of the minimal annihilating polynomial, proving that it is bounded by the product of the degrees of the input polynomials. This bound was subsequently slightly improved in $[22,4]$. Perron's bound gives us the brute-force approach. 
It reduces the problem of computing annihilating polynomial to solving an exponential sized system of linear equations and this can be done in PSPACE. Thus, PSPACE is the "trivial" complexity upper bound for algebraic independence testing, over any field.

The degree bound on the minimal annihilating polynomial happens to be tight. We can give examples of $n$ quadratic polynomials, such that the degree of their minimal annihilating polynomial is $2^{n}$ [22]. There is a hardness result known [22], that shows that computing even the constant term of the annihilating polynomial is NP-hard, and that annihilating polynomial is not of polynomial size in general, unless the polynomial hierarchy collapses.

It turns out that the decision version, i.e. checking if the polynomials are algebraically independent, is much more efficient over zero or large characteristic, even when the polynomials are succinctly represented as circuits. The key idea is a classical result, known as the Jacobian criterion $[20,4]$. It says that if the characteristic of the field is zero, or large enough (compared to the product of degrees of the given polynomials), then the transcendence degree equals the linear rank of the Jacobian matrix of the polynomials. This leads to a simple randomized poly-time algorithm for checking algebraic independence, as we can get the circuits of the partial derivatives efficiently $[3]$ and then use random evaluations to compute the rank of the Jacobian matrix. This final step of randomized evaluation is possible due to the Schwartz-Zippel-DeMillo-Lipton lemma [36, 6, 40].

One direction of the Jacobian criterion (if the polynomials are algebraically dependent, then their Jacobian matrix is not full rank) holds true for any characteristic. But the converse fails if the characteristic is small compared to the product of the degrees of input polynomials. For example, $x^{p}$ is algebraically independent of $\mathbb{F}_{p}$, yet its derivative vanishes. We remark here that if two algebraically independent polynomials over characteristic $p$ have zero Jacobian, then it does not mean that one of them is a $p$ power. Consider, for example, $\left\{x^{p-1} y, x y^{p-1}\right\}$ over $\mathbb{F}_{p}$ for prime $p>2$.

There are infinitely many input instances (set of polynomials), where the Jacobian criterion fails, i.e. Jacobian vanishes even though the given polynomials are independent. Those instances can be characterized by the notion of inseparable extension, that appears in Galois theory, and is formally defined in Sec.2.1. For example, the field extension $\mathbb{F}_{p}(x) / \mathbb{F}_{p}\left(x^{p}\right)$ has inseparable degree $p$ as that many conjugates of $\sqrt[p]{x^{p}}$ in the splitting field are equal. This is a hard algebraic situation with no good geometric interpretation. Such behavior is absent over zero characteristic fields. So, positive characteristic requires inventing new concepts.

Naturally, we would like to come up with an efficient (randomized poly-time) algorithm over small characteristic. Though the failure of Jacobian criterion over small characteristic is known for long $[12,15]$, owing to the interest in algebraic independence from computer science perspective, several recent papers $[7,22,4]$ posed the complexity status of this problem (whether it is in RP) as an open question. One curious aspect is that this problem is one of the rare ones in computer science where the gap between the known time complexity (EXP) and the conjectured one (RP) is that stark!

Talking about the two degrees. Let us consider a case where Jacobian criterion fails and certifying independence gets tricky. Let $m_{1} m_{2}$ be coprime to $p$, and $f_{1}=x_{1}^{p m_{1}}, f_{2}=x_{2}^{m_{2}}$. It is easy to deduce that the degree of the extension $\mathbb{F}_{p}\left(x_{1}, x_{2}\right) / \mathbb{F}_{p}\left(f_{1}, f_{2}\right)$ is $p m_{1} m_{2}$. In fact, the degree of the annihilating polynomial of $\left\{x_{1}, f_{1}, f_{2}\right\}$ (resp. $\left\{x_{2}, f_{1}, f_{2}\right\}$ ) is $p m_{1}$ (resp. $m_{2}$ ). However, the inseparable degree of the extension is only $p$, as the former annihilating polynomial (i.e. $y_{1}^{p m_{1}}-y_{2}$ ) is a polynomial in $y_{1}^{p}$ but not in $y_{1}^{p^{2}}$. Thus, there are cases when the inseparable degree can be much smaller, even $O(1)$, compared to the extension degree. Notice that, in general, the inseparable degree is a $p$-power that divides the extension 
degree, which in turn is upper bounded by $\prod_{i} \operatorname{deg}\left(f_{i}\right)$ (by Perron's bound)- usually an exponentially large parameter. The methods developed in this work only depend on the underlying inseparable degree, thus, our algorithm is expected to be much better than brute-force (in many cases).

A criterion that works for all characteristic for a natural problem like testing algebraic independence would be mathematically interesting. Computational implications of an efficient Jacobian like criterion would include a possible generalization (to small characteristic) of PIT or lower bound results [1], and algebraic extractors or entropy concepts [7].

Work done in case of finite fields. [29] gave a criterion that works over all fields, which they named Witt-Jacobian criterion. One key idea of the Witt-Jacobian criterion is to lift the input polynomials from characteristic $p \geq 2$ to a field of $p$-adics, which is zero characteristic. Witt-Jacobian polynomial can be seen as a scaled up $p$-adic lift of Jacobian polynomial and the criterion involves checking certain monomials (degeneracy testing; which looks hard) rather than zero testing. The main object underlying the proof is the de Rham-Witt pro-complex; a tool from modern algebraic-geometry (an excellent survey is [18]).

Witt-Jacobian criterion improved the complexity of independence testing problem, over positive characteristic, from PSPACE to NP\#P. In the hierarchy of complexity classes, NP\#P is far above RP; thus there is a huge gap between what we have and what we want.

Partial derivative (defined as formal operators on polynomials), that played a key role in Jacobian criterion, behaves strangely over positive characteristic. Though it satisfies the usual rules of derivatives like linearity, product rule and chain rule, one important difference here is the fact that a non-constant polynomial can have a zero derivative. Another difference is that the higher derivatives of order $k \geq p$ are zero for all polynomials over characteristic $p$. Hasse-Schmidt derivatives are variants of usual derivatives, that were originally defined by [17], and independently by [38], to tackle this problem. In computer science literature, Hasse derivatives were used recently in coding theory (see [10] and the references therein), and PIT or lower bounds via generalized versions of shifted partial derivatives [14, 13].

Background on PIT and circuit lower bounds. The problems of derandomization of PIT and proving lower bounds, for explicit family of polynomials, are two fundamental questions in complexity theory. The question of PIT asks to test whether the polynomial computed by an arithmetic circuit is identically zero. This question can be studied in two settings. In the whitebox setting we are allowed to see inside the circuit, whereas in the blackbox setting we can only evaluate the circuit at some field points. The problem of blackbox PIT is equivalent to the problem of designing hitting-sets efficiently. Hitting-set is defined as follows. Let $\mathcal{C}$ be a class of polynomials in $N$ variables over a field $\mathbb{F}$. Then, a set $\mathcal{H} \subseteq \mathbb{F}^{N}$ is called a hitting-set for the class $\mathcal{C}$, if for every nonzero polynomial $C \in \mathcal{C}$, there exists an $x \in \mathcal{H}$ such that $C(x) \neq 0$. PIT has a randomized poly-time algorithm, thanks to Schwartz-ZippelDeMillo-Lipton lemma $[36,40,6]$. Derandomization of PIT is an outstanding open question in complexity theory with several implications, including proving arithmetic circuit lower bounds (refer to [2] \& the survey [37]).

In the world of arithmetic complexity, we have strong structural results like depth reductions $[16,2]$. These results show that strong enough lower bound, or PIT, results for homogeneous depth-4 (or general depth-3) circuits would give us exponential lower bounds and quasipoly-time derandomized PIT for general circuits (up to VP). Recent years have seen a fast growth in papers giving lower bound and PIT results for several special cases of small depth arithmetic circuits [34, 35]. Although there are strong (almost exponential, 
$[26,23])$ lower bounds for homogeneous depth-4 circuits, the best known lower bounds for non-homogeneous depth-4 circuits are only superlinear (see [33] \& the references therein).

Circuits with locally low algebraic rank. Kumar \& Saraf [27] defined a locally low algebraic rank circuit of degree $n$ in $N$ variables over $\mathbb{F}$, denoted $\Sigma \Gamma^{(k)} \Sigma \Pi^{d}$, as: $C=\sum_{i \in[T]} \Gamma_{i}\left(Q_{i 1}, \ldots, Q_{i t}\right)$, where $Q_{i j}$ is a sparse polynomial (all monomials are given explicitly) of degree at most $d$, algRank of $\left\{Q_{i j} \mid j \in[t]\right\}$ is at most $k$, and $\Gamma_{i}$ is an arbitrary $t$-variate polynomial, for $i \in[T]$.

The size of $C$ comprises $N, n, T$ and the maximum sparsity of $Q_{i j}$ 's. Note that $k \leq N$, and we will be interested in the cases when $k d$ is somewhat restricted.

Interestingly, $\Sigma \Gamma^{(n)} \Sigma \Pi$ subsumes homogeneous depth-4 circuits computing a degree $n$ polynomial, as for homogeneous circuits $k \leq t \leq n$ and $\Gamma_{i}$ is merely the product gate. Since this class includes non-homogeneous circuits as well (where $t$ can be arbitrarily larger than $k, n)$, it can be seen as a significant generalization of homogeneous depth- 4 .

This model subsumes certain other interesting models that were studied by $[14,1,4]$ in the context of lower bounds and PIT. Invariably, their methods need to assume that $\mathbb{F}$ has characteristic zero or exponentially large (since partial derivatives are involved). Our goal in this paper is to overcome such restrictions.

\subsection{Our contribution and relation with previous works}

Broadly, in this paper, we prove two main technical theorems, one about the algebraically dependent polynomials and the other about algebraically independent polynomials. We apply these two theorems to obtain an algebraic independence testing algorithm, an arithmetic circuit lower bound over arbitrary field and a PIT algorithm (over fields of characteristic larger than the individual-degree of the polynomial). We now describe each of the results.

Algebraic dependence to approximate functional dependence. We show that over arbitrary fields, algebraic dependence of polynomials $f_{1}, \ldots, f_{m}$ imply the existence of a transcendence basis such that all the polynomials $f_{1}, \ldots, f_{m}$ can be obtained (upto a random shift and a truncation) as a polynomial function of the basis elements (Thm.10). Essentially, to obtain the desired polynomial, say $f_{k}$, we truncate a polynomial function in the elements of the basis upto the degree of $f_{k}$. This generalizes the functional dependence result of [27, Lem.3.1] which asserted the same over fields of zero (or large) characteristic.

We use a proof approach which is different from [27] to achieve the more general results. In the case of fields of zero characteristic, the subtle strength that this functional dependence property possesses is that any transcendence basis serves the purpose, which in general is false over positive characteristic. Our result explains this subtlety using the concept of separating transcendence basis from Galois theory (Sec.2.1). With this, a simple algebraic manipulation on the annihilating polynomial, and subspace of polynomial products (Lem.12), yields a functional dependence up to any desired degree of approximation. (This is a bit simpler than the approach of [27, Lem.2.4] where they approximate the roots of any multivariate polynomial using [11, Lem.3.1]. Such methods also appear in classical analysis under Implicit Function Theorems, see [25].)

Eg. $\left\{x_{1}, x_{2}, x_{1} x_{2}^{2}\right\}$ are algebraically dependent over $\overline{\mathbb{F}}_{2}$. Pick random field elements $a_{1}, a_{2}$. The shifted polynomials are $\left\{x_{1}+a_{1}, x_{2}+a_{2},\left(x_{1}+a_{1}\right)\left(x_{2}^{2}+a_{2}^{2}\right)\right\}$. Clearly, $\left(x_{2}+a_{2}\right)$ is not a function of the other two modulo the ideal $\langle\mathbf{x}\rangle^{2}$. However, $\left(x_{1}+a_{1}\right)$ is trivially a function of the other two, namely, $\left(x_{1}+a_{1}\right) \equiv a_{2}^{-2} \cdot\left(x_{1}+a_{1}\right)\left(x_{2}^{2}+a_{2}^{2}\right) \bmod \langle\mathbf{x}\rangle^{2}$. 
Algebraically independent polynomials - Criterion. The above example shows that over fields of positive characteristic, an approximate functional dependence may exist even in the case of algebraically independent polynomials. We overcome this issue and show that the independence can be captured by truncating the polynomial function in the basis elements upto a precise parameter, i.e. if we choose the truncation point to be greater than that parameter, then algebraically independent polynomials cannot exhibit functional dependence (Thm.13). This parameter is actually the inseparable degree of an appropriate field extension, which is a well studied concept in Galois theory (Sec.2.1).

Continuing the above example- $\left\{x_{1}, x_{1} x_{2}^{2}\right\}$ are algebraically independent over $\overline{\mathbb{F}}_{2}$. Pick random field elements $a_{1}, a_{2}$. The shifted polynomials are $\left\{x_{1}+a_{1},\left(x_{1}+a_{1}\right)\left(x_{2}^{2}+a_{2}^{2}\right)\right\}$. It can be verified that neither is a polynomial function of the other modulo the ideal $\langle\mathbf{x}\rangle^{3}$. This becomes a certificate of algebraic independence. (Note that the inseparable degree of $\mathbb{F}_{2}\left(x_{1}, x_{2}\right) / \mathbb{F}_{2}\left(x_{1}, x_{1} x_{2}^{2}\right)$ is 2 .)

When the inseparable degree is 1 (which means a separable extension), then looking at the truncation upto the linear term of shifted basis elements would suffice. So, our result implies that separable extension is precisely the case when the Jacobian works (an exposition can be found in the full version). For higher inseparable degree $t$, our result can be reinterpreted as giving a Jacobian like result: algebraically independent polynomials have $\mathbb{F}(\mathbf{z})$-linearly independent higher differentials (Sec.2.2), modulo a carefully chosen subspace $\mathcal{U}_{t}$ (Rmk.11). This follows by considering the Taylor series, around a "generic" point $\mathbf{z}$, whence, the functional independence of polynomials shifted by $\mathbf{z}$, implies the linear independence of shifted polynomials modulo $\mathcal{U}_{t}$. As shifted polynomials contain all the Hasse-Schmidt higher derivatives (wrt $\mathbf{x}$ and evaluated at the point $\mathbf{z}$ ), we deduce their $\mathbb{F}(\mathbf{z})$-linear independence modulo $\mathcal{U}_{t}$.

Again, a key technical lemma used in finishing the proof is Lem.12 (subspace reduction), which concerns the ideal theoretic properties of the subspace $\mathcal{U}_{t}$. Basically, it helps us prove that if $\left\{h_{1}, \ldots, h_{n}\right\}$ are polynomials with their degree $(\leq t)$-part having algebraically independent leading monomials, and $g_{n}$ functionally depends on $\left\{g_{1}, \ldots, g_{n-1}\right\}$ (with truncation beyond $t$ ), then some $h_{i}$ is functionally independent of $\left\{g_{1}, \ldots, g_{n}\right\}$.

Application 1: Testing algebraic independence. An easy consequence of Thm.10 and Thm.13 is that we get a randomized poly-time algorithm for testing algebraic independence of polynomials over finite fields (say, $\mathbb{F}_{q}$ of characteristic $p$ ) in the cases when the inseparable degree is constant. Since the latter is a p-power (Sec.2.1), our algorithm is interesting when $p$ is a constant. (Whenever required, we can assume wlog that the input is $n$ circuits in $n$ variables over an algebraically closed field; see full version for simple proofs.)

- Theorem 1 (Independence testing). For circuits $\mathbf{f} \in \mathbb{F}_{q}[\mathbf{x}]$, we have a randomized poly $(s$, $\left.\left(\begin{array}{c}t+n \\ n\end{array}\right)\right)$-time algebraic independence testing algorithm, where the inseparable degree of the field extension $\mathbb{F}_{q}(\mathbf{x}) / \mathbb{F}_{q}(\mathbf{f})$ is $t$ (assuming $\mathbf{f}$ algebraically independent) \& input size is $s$.

This covers a lot of interesting cases as the inseparable degree can be quite small even in case of polynomials with exponential degree. As a simple example, take two bivariate circuits of exponential degree over $\mathbb{F}_{2}$. Suppose they are independent and their Jacobian is nonzero. Now if we square any one of these two, then Jacobian would fail as the inseparable degree becomes 2. Previously known algorithms cannot deal with even such a simple case, whereas we easily handle the case by trying our test with $t=2$. In general, the inseparable degree is upper bounded by Perron's degree bound (product of degrees of given polynomials, [32]), so in the worst-case our algorithm is exponential-time. (Witt-Jacobian criterion [29] 
is exponential-time in all cases.) We illustrate the overall idea, and its comparison with Jacobian criterion, in the figure in the conclusion (Sec.4).

An interesting by-product of the algorithm is that it computes the inseparable degree, of the given independent polynomials, in the same time.

Application 2: Lower bound for locally low algebraic rank circuits. Using the functional dependence result, we give an explicit family of polynomials in VNP of degree $n$ in $N$ variables, where $N=n^{O(1)}$ such that any $\Sigma \Gamma^{(n)} \Sigma \Pi$ circuit computing it has size $N^{\Omega(\sqrt{n})}$. We obtain this lower bound over arbitrary fields. This generalizes the lower bound of [27, Thm.1.4] which itself was a strong generalization of the shifted partials based homogeneous depth-4 lower bounds [23] and Jacobian based lower bounds [1] (all over zero or large characteristic). Since our functional dependence generalizes the key technical ingredient of [27] to arbitrary fields, we are able to get the same lower bound (for the same model and hard polynomial family) over arbitrary fields. Formally,

Theorem 2. Let $\mathbb{F}$ be any field. There exists a family $\left\{P_{n}\right\}$ of polynomials in VNP, such that $P_{n}$ is a polynomial of degree $n$ in $N=n^{O(1)}$ variables with 0,1 coefficients, and for any $\Sigma \Gamma^{(k)} \Sigma \Pi$ circuit $C$, if $k \leq n$ and if $C$ computes $P_{n}$ over $\mathbb{F}$, then $\operatorname{Size}(C) \geq N^{\Omega(\sqrt{n})}$.

- Remark. As remarked by [27], the above model is challenging even for $k=2$ (\& was open before us for small characteristic fields). Also, the proof goes through for any $k=n^{O(1)}$, as long as one picks $N$ as an appropriately large polynomial in $n$.

The proof of this theorem closely follows [27], and is sketched in the full version.

Application 3: Hitting-set for $\boldsymbol{\Sigma} \boldsymbol{\Gamma}^{(\boldsymbol{k})} \boldsymbol{\Sigma} \boldsymbol{\Pi}^{\boldsymbol{d}}$ circuits. We show that for any size- $s$ circuit $C \in \Sigma \Gamma^{(k)} \Sigma \Pi^{d}$, where $k, d=\operatorname{polylog}(s)$, over fields of characteristic $p>$ individual-degree $(C)$, there exists a quasipoly $(s)$-time hitting-set.

Theorem 3. Let $\mathbb{F}$ be any field of characteristic $p$. There exists an $\exp \left(\log ^{O(1)} s\right)$-time constructible hitting-set $\mathcal{H} \subseteq \overline{\mathbb{F}}^{N}$ for size-s circuit $C \in \Sigma \Gamma^{(k)} \Sigma \Pi^{d}$ with $k d=\log ^{O(1)} s$, assuming $p>$ individual-degree $(C)$ or $p=0$.

Again, the proof follows [27]. For PIT, algebraic rank based models have already been considered by $[4,1,27]$. Our result generalizes some of these results to smaller positive characteristic (only requiring $p>$ individual-degree $(C)$ ). The previous results required $p>d^{k}$, which is super-polynomial in the above regime. Our inability to remove this restriction lies in the nature of shifted partials $[14$, Lem.4.13]. Eg. the dimension of shifted partials of a $p$-power monomial $x_{1}^{p^{e_{1}}} \cdots x_{n}^{p^{e_{n}}}$ is not that large over $\mathbb{F}_{p}$.

\section{Preliminaries: Jacobi, Galois and Hasse-Schmidt}

We define the central object related to the testing of algebraic independence is the Jacobian.

- Definition 4 (Jacobian). The Jacobian of polynomials $\mathbf{f}=\left\{f_{1}, \cdots, f_{m}\right\}$ in $\mathbb{F}\left[x_{1}, \cdots, x_{n}\right]$ is the matrix $\mathcal{J}_{\mathbf{x}}(\mathbf{f})=\left(\partial_{x_{j}} f_{i}\right)_{m \times n}$, where $\partial_{x_{j}} f_{i}:=\partial f_{i} / \partial x_{j}$.

We state the classical Jacobian criterion $[20,4]$.

- Lemma 5 (Jacobian criterion). Let $\mathbf{f} \subset \mathbb{F}[\mathbf{x}]$ be a finite set of polynomials of degree at most $d$, and $\operatorname{trdeg}_{\mathbb{F}} \mathbf{f} \leq r$. If $\operatorname{char}(\mathbb{F})=0$, or $\operatorname{char}(\mathbb{F})>d^{r}$, then $\operatorname{trdeg}_{\mathbb{F}} \mathbf{f}=\operatorname{rank}_{\mathbb{F}(\mathbf{x})} \mathcal{J}_{\mathbf{x}}(\mathbf{f})$. 
Previously, we saw some examples of polynomials over fields of smaller characteristic where the Jacobian fails. Here is another nontrivial example: $\mathbf{f}=\left\{x_{1}^{2} x_{2}+x_{1}^{3}, x_{1} x_{2}^{2}+x_{1} x_{2}^{5}\right\}$ in $\mathbb{F}_{3}\left[x_{1}, x_{2}\right]$ is a set of algebraically independent polynomials, but $\operatorname{rank}_{\mathbb{F}_{3}(\mathbf{x})} \mathcal{J}_{\mathbf{x}}(\mathbf{f})=1$, and hence the criterion fails.

\subsection{Inseparability \& separating transcendence basis}

For this section, let $\mathbb{E} \supseteq \mathbb{F}$ be fields. Failure of the Jacobian criterion can be explained using the fundamental concept of inseparability from Galois theory [19].

- Definition 6. An $f \in \mathbb{F}[x]$ is separable if it has no multiple roots in its splitting field.

It is easy to prove that. For an irreducible $f$, separability is implied by the non-zeroness of $\partial_{x} f$. Thus, if $\operatorname{char}(\mathbb{F})=0$, then any irreducible polynomial is separable. It further implies that if $\operatorname{char}(\mathbb{F})=p>0$ then, an irreducible $f$ is separable if and only if $f \notin \mathbb{F}\left[x^{p}\right]$. We have this notion of separability in case of field extensions as well. An algebraic extension $\mathbb{E} / \mathbb{F}$ is said to be separable if every element $\alpha \in \mathbb{E}$ has a minimal polynomial over $\mathbb{F}$ that is separable.

For polynomials $f_{1}, \ldots, f_{m} \in \mathbb{F}\left[x_{1}, \ldots, x_{n}\right]$, we deal with the extension $\mathbb{F}\left(x_{1}, \ldots, x_{n}\right) /$ $\mathbb{F}\left(f_{1}, \ldots, f_{m}\right)$. This extension is algebraic iff $\operatorname{trdeg}(\mathbf{f})=n$ (as every $x_{j}$ depends on $\left.\mathbf{f}\right)$. In which case, the extension $\mathbb{F}(\mathbf{x}) / \mathbb{F}(\mathbf{f})$ is separable iff the minimal polynomial of $x_{j}$ over $\mathbb{F}(\mathbf{f})$ is separable, for all $j \in[n]$. The latter, clearly, is the case when $\operatorname{char}(\mathbb{F})=0$. When $\operatorname{char}(\mathbb{F})$ $=p>0$, the extension is inseparable if there exists $j \in[n]$, such that the minimal polynomial of $x_{j}$ over $\mathbb{F}(\mathbf{f})$ lives in $\mathbb{F}(\mathbf{f})\left[y^{p}\right]$. Thus for every $x_{j}$, we have an $m_{j}$ such that $x_{j}^{p^{m_{j}}}$ has a separable minimal polynomial over $\mathbb{F}(\mathbf{f})$.

The inseparable degree of the extension $\mathbb{F}(\mathbf{x}) / \mathbb{F}(\mathbf{f})$ is defined as the minimum $p^{m}$ such that the minimal polynomial of $x_{j}^{p^{m}}$ over $\mathbb{F}(\mathbf{f})$ is separable, for all $j \in[n]$. We also associate this inseparable degree with the set $\mathbf{f}$.

In the case when $\mathbf{f}$ are algebraically dependent, we would like to use a "good" transcendence basis. This is captured by:

- Definition 7 (Separating transcendence basis). A field extension $\mathbb{E} / \mathbb{F}$ is called separably generated if there exists an algebraically independent set (i.e. transcendence basis) $S=$ $\left\{f_{1}, \ldots, f_{r}\right\} \subset \mathbb{E}$ such that $\mathbb{E} / \mathbb{F}(S)$ is algebraic and separable.

$S$ is called a separating transcendence basis of $\mathbb{E} / \mathbb{F}$.

It is a classical result that such bases exist for fields that we are interested in.

- Theorem 8. Consider a finite set of polynomials $\mathbf{f} \subset \mathbb{F}[\mathbf{x}]$. If $\mathbb{F}$ is a finite field (resp. an algebraically closed field) then there exists a separating transcendence basis, of $\mathbb{F}(\mathbf{f}) / \mathbb{F}$, in $\mathbf{f}$.

In case $\mathbb{F}$ is a zero characteristic field then every transcendence basis of $\mathbf{f}$ is a separating one of the extension $\mathbb{F}(\mathbf{f}) / \mathbb{F}$.

Proof. It is clear that if $\mathbb{F}$ has characteristic zero then there is no possibility of inseparability.

Let $\mathbb{F}$ be a finite (resp. algebraically closed) field. [24, Thm.7.20] shows that the extension $\mathbb{F}(\mathbf{f}) / \mathbb{F}$ is separably generated. Furthermore, $[24$, Thm.7.18] shows that $\mathbf{f}$ contains a subset that is a separating transcendence basis of the extension.

Examples. Extension $\mathbb{F}_{3}\left(x^{3}\right) / \mathbb{F}_{3}$ has $\left\{x^{3}\right\}$ as a separating transcendence basis. Consider the two transcendence bases of the extension $\mathbb{F}_{3}\left(x^{2}, x^{3}\right) / \mathbb{F}_{3}-\left\{x^{3}\right\}$ and $\left\{x^{2}\right\}$. The latter is a separating transcendence basis, but the former is not. 


\subsection{Taylor expansion at $\mathrm{z}$, higher derivatives \& differentials}

We consider the application of shift (or translation) to our polynomials. We view this as writing the Taylor expansion of a polynomial $f(\mathbf{x})$ at a "generic" point $\mathbf{z}$ [13, Sec.C.1]. A second view is that of computing the Hasse-Schmidt higher derivatives of $f$ at the point $\mathbf{z}$ $[14,10]$. A third view is seeing the shifted polynomial as a Hasse-Schmidt differential [39]. We collect these equivalent viewpoints in a single definition.

- Definition 9 (Formal shift). We see $f(\mathbf{x}+\mathbf{z})$ as a polynomial in $R:=\mathbb{F}_{p}(\mathbf{z})[\mathbf{x}]$ where the variables $x_{1}, \ldots, x_{n}$ are shifted respectively by the function field elements $z_{1}, \ldots, z_{n}$.

Now the coefficient of $m:=x_{1}^{\ell_{1}} \cdots x_{n}^{\ell_{n}}$ in the Taylor-series expansion of $f(\mathbf{x}+\mathbf{z})$ can be

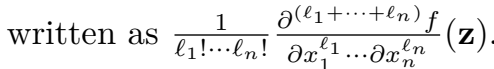

This is called the Hasse-Schmidt derivative of $f$ wrt $m$ evaluated at the point $\mathbf{z}$. It can be denoted, by some abuse of notation, as $\left.\partial_{m} f(\mathbf{x})\right|_{\mathbf{z}}$.

Finally, we can see the formal shift as a Hasse-Schmidt differential, namely, $f(\mathbf{x}+\mathbf{z})=$ $\left.\sum_{m} m \cdot \partial_{m} f(\mathbf{x})\right|_{\mathbf{z}}$ (sum over all monomials $m$ in $\mathbf{x}$ ).

Example. We have $\partial^{2} x^{2} / \partial x^{2}=0$ over $\mathbb{F}_{2}$, but $\partial^{2} x^{2} / 2 ! \partial x^{2}=1$. Thus, Hasse-Schmidt derivatives offer a natural solution to this vanishing problem.

This connection between the shifts and Hasse-Schmidt higher derivatives/ differentials is what motivated us to search for the right framework to study algebraic independence.

Now the Jacobian criterion is given in terms of the first order derivatives of the polynomials and the failure of Jacobian essentially exposes the inability of first order derivative in capturing independence. Intuitively, it seems that going to higher derivatives may help. The above connection points out that perhaps we need to look at higher degree terms (wrt $\mathbf{x})$ of $f(\mathbf{x}+\mathbf{z})$ to get an algebraic independence criterion in cases where Jacobian fails. Eventually, we will see that the intuition is indeed true.

Operator $\mathcal{H}$. For notational convenience, we define the non-constant part of $f(\mathbf{x}+\mathbf{z})$ up to degree $\leq t$ wrt $\mathbf{x}$, as $\mathcal{H}_{t} f:=f^{\leq t}(\mathbf{x}+\mathbf{z})-f(\mathbf{z})$.

This is easier to work with when we do manipulations modulo the ideal $\langle\mathbf{x}\rangle_{R}^{t+1}$.

\section{Main structure theorems}

We use the following standard notation in the paper:

1. $\mathbb{F}$ is an arbitrary field. $\overline{\mathbb{F}}$ is its algebraic closure.

2. $\mathbb{F}_{q}$ is a finite field of size $q$ and characteristic $p \geq 2$.

3. Let $R \supseteq S$ be a commutative ring extension over a field $\mathbb{F}$, let $v_{1}, \ldots, v_{m} \in R$ and $r \geq 1$. Then $\left\langle v_{1}, \ldots, v_{m}\right\rangle_{S}^{r}$ is simply the set of all $S$-linear combinations of products $v_{i_{1}} \cdots v_{i_{r}}$ $\left(i_{j}\right.$ 's in $\left.[m]\right)$. It is both an $S$-module and an $\mathbb{F}$-vector space. (It is an ideal when $R=S$.)

4. For a polynomial $h \in \mathbb{F}[\mathbf{x}], h^{\leq d}$ extracts out the degree $\leq d$ part of $h$ and returns it as an element in $\mathbb{F}[\mathbf{x}]$ again.

5. For a polynomial $h \in \mathbb{F}[\mathbf{x}], h^{[\leq d]}$ extracts out the degree $\leq d$ part of $h$ and returns it as a $d+1$ tuple, where for $i \in[0 \ldots d]$, $i$-th entry of the tuple contains $h^{=i}$ which is defined as the homogeneous component of $h$ of degree $i$. 


\subsection{Functional dependence for algebraically dependent polynomials}

A fact about linear independence is that if $f_{1}, \ldots, f_{m} \in \mathbb{F}[\mathbf{x}]$ are linearly dependent, it also implies that every polynomial can be written as a linear combination of the polynomials in the basis. The question is whether the same can be extended to algebraic dependence: Does algebraic dependence imply that all the polynomials can be written as a function of the polynomials in the transcendence basis? It was shown in [27, Lem.3.1] that it is indeed true (approximately) over fields of zero (and large) characteristic.

We generalize the property using a different proof approach and show that algebraic dependence implies functional dependence over arbitrary fields (to arbitrary degree of approximation $t$ ).

- Theorem 10 (Functional dependence over arbitrary fields). Let $\mathbf{f}=\left\{f_{1}, \ldots, f_{m}\right\} \subset \mathbb{F}\left[x_{1}, \ldots\right.$, $\left.x_{n}\right]$ be a set of polynomials, where $\mathbb{F}$ is any field, and $t \in \mathbb{N}$. If trdeg of $\left\{f_{1}, \ldots, f_{m}\right\}$ is $k$, then there exist algebraically independent $\left\{g_{1}, \ldots, g_{k}\right\} \subset \mathbf{f}$, such that for random $\mathbf{a} \in \overline{\mathbb{F}}^{n}$, there are polynomials $h_{i} \in \overline{\mathbb{F}}\left[Y_{1}, \ldots, Y_{k}\right]$ satisfying, $\forall i \in[m], f_{i}^{\leq t}(\mathbf{x}+\mathbf{a})=$ $h_{i}^{\leq t}\left(g_{1}(\mathbf{x}+\mathbf{a}), \ldots, g_{k}(\mathbf{x}+\mathbf{a})\right)$.

- Remark. Clearly, $\overline{\mathbb{F}}^{n}$ is an infinite space. What we mean here by a random a is "random point in any sufficiently large, but finite, subset of the space". It will be clear from the proof that it would suffice to sample from any set of size at most exponential in the input size. We skip the detailed estimate as in this paper merely existence of $\mathbf{a}$ is needed.

We will use $\mathbf{z}$ as a formal variable ( $n$-tuple) and can fix it later to a suitable constant a. To prove the theorem, we consider the $\operatorname{ring} R:=\overline{\mathbb{F}}(\mathbf{z})[\mathbf{x}]$ and its ideal $\mathcal{I}_{0}:=\langle\mathbf{x}\rangle_{R}$. The ideal collects the non-constant linear polynomials. Now, define the ideal $\mathcal{I}_{t}:=\mathcal{I}_{0}^{t+1}$ and the quotient algebra $\mathcal{Q}_{t}:=R / \mathcal{I}_{t}$, i.e. we are filtering out, or truncating, all the terms of degree $>t$. Now $\mathcal{Q}_{t}$ can also be seen as a finite $\left(\begin{array}{c}n+t \\ n\end{array}\right)$ dimensional vector space over $\overline{\mathbb{F}}(\mathbf{z})$ whose basis is monomials in $\mathbf{x}$ of degree at most $t$. In our theorems and proofs, most of the operations happen in this quotient ring $\mathcal{Q}_{t}$ for increasing $t$ 's.

In our analysis, we plan to use the shifting of the variables in the evaluated annihilating polynomial of $\left\{f_{i}, g_{1}, \ldots, g_{k}\right\}$, and it is clear that on applying the shifts, we will end up having terms of the form $\left(\mathcal{H}_{t} f_{i}\right)^{j_{0}}\left(\mathcal{H}_{t} g_{1}\right)^{j_{1}} \cdots\left(\mathcal{H}_{t} g_{k}\right)^{j_{k}}$ (recall that in $Q_{t}, f(\mathbf{x}+\mathbf{z})=f(\mathbf{z})+\mathcal{H}_{t} f(\mathbf{x})$ ). Now, note that due to the filtration in $\mathcal{Q}_{t}$, some of these terms will be equivalent to terms involving $\mathcal{H}_{r}$ with $r<t$. We consider an appropriate subspace $\mathcal{U}_{t} \subset \mathcal{Q}_{t}$ generated by such "higher" products, which we formally define as: $\mathcal{U}_{1}:=\{0\}$ and

$$
\mathcal{U}_{t}:=\left\langle\mathcal{H}_{t-1} f_{i}, \mathcal{H}_{t-1} g_{1}, \ldots, \mathcal{H}_{t-1} g_{k}\right\rangle_{\mathbb{F}(\mathbf{z})}^{2}+\cdots+\left\langle\mathcal{H}_{1} f_{i}, \mathcal{H}_{1} g_{1}, \ldots, \mathcal{H}_{1} g_{k}\right\rangle_{\mathbb{F}(\mathbf{z})}^{t}, \quad t \geq 2 .
$$

- Remark 11. In $\mathcal{Q}_{t}$, observe that, this is the same subspace as $\left\langle\mathcal{H}_{t} f_{i}, \mathcal{H}_{t} g_{1}, \ldots, \mathcal{H}_{t} g_{k}\right\rangle_{\mathbb{F}(\mathbf{z})}^{2}+\cdots$ $+\left\langle\mathcal{H}_{t} f_{i}, \mathcal{H}_{t} g_{1}, \ldots, \mathcal{H}_{t} g_{k}\right\rangle \frac{t}{\bar{F}(\mathbf{z})}$

Proof of Theorem 10. Consider the set $\mathbf{f}:=\left\{f_{1}, \ldots, f_{m}\right\} \subset \mathbb{F}[\mathbf{x}]$ with algebraic rank $k$. If we work over $\overline{\mathbb{F}}$, then Thm.8 guarantees the existence of a separating transcendence basis $\left\{g_{1}, \ldots, g_{k}\right\} \subseteq \mathbf{f}$. Let $g_{0}:=f_{i}$ for a fixed $i \in[m]$. Now we consider the separable annihilating polynomial $A(\mathbf{y})=\sum_{\mathbf{e}_{\ell}} a_{\mathbf{e}_{\ell}} \mathbf{y}^{\mathbf{e}_{\ell}}$ of the set $\mathbf{g}:=\left\{g_{0}, g_{1}, \ldots, g_{k}\right\}$, and $a_{\mathbf{e}_{\ell}}$ 's are in $\overline{\mathbb{F}}$ ( $\mathbf{e}_{\ell}$ is a $(k+1)$-tuple $\left.\left(e_{j \ell} \mid j \in[0 \ldots k]\right)\right)$. Thus, $A(\mathbf{g})=\sum_{\mathbf{e}_{\ell}} a_{\mathbf{e}_{\ell}} \prod_{j=0}^{k} g_{j}(\mathbf{x})^{e_{j \ell}}=0$. We now apply the formal shift $\mathbf{x} \mapsto \mathbf{x}+\mathbf{z}$ to get $A\left(g_{0}(\mathbf{x}+\mathbf{z}), \ldots, g_{k}(\mathbf{x}+\mathbf{z})\right)=0$, i.e. $\sum_{\mathbf{e}_{\ell}} a_{\mathbf{e}_{\ell}} \prod_{j} g_{j}(\mathbf{x}+\mathbf{z})^{e_{j \ell}}=$ 0 .

We now study this relation in the algebra $\mathcal{Q}_{t}$. By Taylor series expansion, we know that $f(\mathbf{x}+\mathbf{z}) \equiv f(\mathbf{z})+\mathcal{H}_{t} f(\mathbf{x})$ in $\mathcal{Q}_{t}$, so we get $\sum_{\mathbf{e}_{\ell}} a_{\mathbf{e}_{\ell}} \prod_{j}\left(g_{j}(\mathbf{z})+\mathcal{H}_{t} g_{j}\right)^{e_{j \ell}} \equiv 0$. The binomial 
expansion gives a compact expression:

$$
\sum_{\mathbf{e}_{\ell}} a_{\mathbf{e}_{\ell}} \sum_{\mathbf{0} \leq \mathbf{s} \leq \mathbf{e}_{\ell}}\left(\begin{array}{c}
\mathbf{e}_{\ell} \\
\mathbf{s}
\end{array}\right) \cdot\left(\mathcal{H}_{t} \mathbf{g}\right)^{\mathbf{s}} \cdot \mathbf{g}^{\mathbf{e}_{\ell}-\mathbf{s}} \equiv 0 .
$$

Note that the contribution by $\mathbf{s}=\mathbf{0}$ terms sum up to $\sum_{\mathbf{e}_{\ell}} a_{\mathbf{e}_{\ell}} \prod_{j=0}^{k} g_{j}(\mathbf{z})^{e_{j \ell}}$ which is zero. This implies that an $\overline{\mathbb{F}}(\mathbf{z})$-linear combination of the products of the form $\left(\mathcal{H}_{t} g_{0}\right)^{s_{0}} \cdots\left(\mathcal{H}_{t} g_{k}\right)^{s_{k}}$, $\sum_{j} s_{j} \geq 1$, vanishes in $\mathcal{Q}_{t}$. Now the key step is to separate out the terms linear in $\mathcal{H}_{t} g_{j}$ and switch the sums, to obtain

$$
\begin{aligned}
\mathcal{H}_{t} g_{0} \cdot g_{0}(\mathbf{z})^{-1}\left(\sum_{\mathbf{e}_{\ell}} a_{\mathbf{e}_{\ell}} \cdot e_{0 \ell} g_{0}^{e_{0 \ell}} \cdots g_{k}^{e_{k \ell}}\right) & +\sum_{j \in[k]} \mathcal{H}_{t} g_{j} \cdot g_{j}(\mathbf{z})^{-1}\left(\sum_{\mathbf{e}_{\ell}} a_{\mathbf{e}_{\ell}} \cdot e_{j \ell} g_{0}^{e_{0 \ell}} \cdots g_{k}^{e_{k \ell}}\right) \\
& +\left(\text { higher terms with } \sum_{j} s_{j} \geq 2\right) \equiv 0 .
\end{aligned}
$$

Further, we argue using the minimality and separability of $A$ (in terms of the first variable) that the "linear" term $\mathcal{H}_{t} g_{0}$ in the vanishing sum above has a non-zero coefficient: as it would either mean a lower degree annihilating polynomial $A:=\sum_{\mathbf{e}_{\ell}} a_{\mathbf{e}_{\ell}} e_{0 \ell} y_{0}^{e_{0 \ell}-1} \cdot y_{1}^{e_{1 \ell}} \cdots y_{k}^{e_{k \ell}}$ i.e. contradicting the minimality, or that all the $e_{0 \ell}$ 's are divisible by $p$ (when $\mathbb{F}$ has characteristic $p$ ) which means that $f_{i}$ does not depend separably on $\left\{g_{1}, \ldots, g_{k}\right\}$; which contradicts the fact that $\left\{g_{1}, \ldots, g_{k}\right\}$ is a separating transcendence basis.

Thus, we get that $\mathcal{H}_{t} g_{0}$ lives in the $\overline{\mathbb{F}}(\mathbf{z})$-linear span of $\mathcal{H}_{t} g_{1}, \ldots, \mathcal{H}_{t} g_{k}$ modulo the subspace generated by the higher terms of the summation in Eqn.1. So, $\mathcal{H}_{t} g_{0}$ lives in the $\overline{\mathbb{F}}(\mathbf{z})$-linear span of $\mathcal{H}_{t} g_{1} \ldots, \mathcal{H}_{t} g_{k}$ modulo the subspace $\mathcal{U}_{t}(\mathrm{Rmk} .11)$ in $\mathcal{Q}_{t}$.

We got $\mathcal{H}_{t} f_{i} \in\left\langle\mathcal{H}_{t} g_{1}, \ldots, \mathcal{H}_{t} g_{k}\right\rangle_{\overline{\mathbb{F}}(\mathbf{z})}+\mathcal{U}_{t}$. Now, we are in a position to apply Lemma 12 , which essentially says that if $\mathcal{H}_{r} f_{n}$ depends on higher order terms (in the sense of Equation 1) then it can be "dropped" from the ideal manipulations. Thus, we get that $\mathcal{H}_{t} f_{i} \in$ $\left\langle\mathcal{H}_{t} g_{1}, \ldots, \mathcal{H}_{t} g_{k}\right\rangle_{\overline{\mathbb{F}}(\mathbf{z})}+\left\langle\mathcal{H}_{t-1} g_{1}, \ldots, \mathcal{H}_{t-1} g_{k}\right\rangle_{\overline{\mathbb{F}}(\mathbf{z})}^{2}+\cdots+\left\langle\mathcal{H}_{1} g_{1}, \ldots, \mathcal{H}_{1} g_{k}\right\rangle_{\overline{\mathbb{F}}(\mathbf{z})}^{t}$. The latter (by Rmk.11) is exactly $\left\langle\mathcal{H}_{t} g_{1}, \ldots, \mathcal{H}_{t} g_{k}\right\rangle_{\overline{\mathbb{F}}(\mathbf{z})}+\left\langle\mathcal{H}_{t} g_{1}, \ldots, \mathcal{H}_{t} g_{k}\right\rangle_{\overline{\mathbb{F}}(\mathbf{z})}^{2}+\cdots+\left\langle\mathcal{H}_{t} g_{1}, \ldots, \mathcal{H}_{t} g_{k}\right\rangle_{\overline{\mathbb{F}}(\mathbf{z})}^{t}$.

This implies $f_{i}(\mathbf{x}+\mathbf{z}) \in\left\langle 1, g_{1}(\mathbf{x}+\mathbf{z}), \ldots, g_{k}(\mathbf{x}+\mathbf{z})\right\rangle_{\mathbb{F}(\mathbf{z})}^{t}$ in $\mathcal{Q}_{t}$, which yields the approximate functional dependence around a generic point $\mathbf{z}$.

Fixing $\mathbf{z}$ (avoiding some bad choices that make certain $\mathbf{z}$-polynomials in the above proof zero) to an element $\mathbf{a} \in \overline{\mathbb{F}}^{n}$ finishes the proof.

We now formally state our subspace reduction lemma:

- Lemma 12 (Subspace reduction). Let $\mathbb{F}$ be any field, $R:=\mathbb{F}(\mathbf{z})[\mathbf{x}], \mathcal{Q}_{r}:=R /\langle\mathbf{x}\rangle^{r+1}$ for $r \geq 1$, and $\mathbf{f} \subset \mathbb{F}[\mathbf{x}]$. Define $\mathcal{U}_{1}=\mathcal{V}_{1}=\{0\}$, and for $u \in\langle\mathbf{x}\rangle_{R}, r \geq 2$, define the subspaces (in the quotient algebra $\mathcal{Q}_{r}$ ),

$$
\begin{aligned}
\mathcal{U}_{r} & :=\left\langle\mathcal{H}_{r-1} f_{1}, \ldots, \mathcal{H}_{r-1} f_{n}\right\rangle_{\mathbb{F}(\mathbf{z})}^{2}+\cdots+\left\langle\mathcal{H}_{1} f_{1}, \ldots, \mathcal{H}_{1} f_{n}\right\rangle_{\mathbb{F}(\mathbf{z})}^{r}, \\
\mathcal{V}_{r}: & =\left\langle\mathcal{H}_{r-1} f_{1}, \ldots, \mathcal{H}_{r-1} f_{n-1}, u\right\rangle_{\mathbb{F}(\mathbf{z})}^{2}+\cdots+\left\langle\mathcal{H}_{1} f_{1}, \ldots, \mathcal{H}_{1} f_{n-1}, u\right\rangle_{\mathbb{F}(\mathbf{z})}^{r} . \\
\text { If } \mathcal{H}_{t} f_{n} & \in\left\langle\mathcal{H}_{t} f_{1}, \ldots, \mathcal{H}_{t} f_{n-1}, u\right\rangle_{\mathbb{F}(\mathbf{z})}+\mathcal{U}_{t}, \text { then } \mathcal{U}_{t} \subseteq \mathcal{V}_{t}(\text { for any } t \in \mathbb{N}) .
\end{aligned}
$$

Remark. If $u=0$ then the lemma "reduces" the $n$ polynomial generators, of the subspace $\mathcal{U}_{t}$, by one. Hence, the name "subspace reduction". A simple inductive proof of the lemma is given in the full version. 


\subsection{Algebraically independent polynomials: Criterion}

Having proved the functional dependence for algebraically dependent polynomials, one naturally asks whether a converse exists (for arbitrary fields? to what degree?). We will characterize this completely.

It's all about the inseparable degree- We show that if $f$ is algebraically independent of $\left\{g_{1}, \ldots, g_{k}\right\}$ then, under a random shift, $f$ cannot be written as a function of $\left\{g_{1}, \ldots, g_{k}\right\}$ when chosen to truncate at (or beyond) the inseparable degree of the extension $\mathbb{F}_{q}(\mathbf{x}) / \mathbb{F}_{q}\left(f, g_{1}\right.$, $\left.\ldots, g_{k}\right)$. Moreover, for each truncation at lower degrees we get functional dependence.

- Theorem 13 (Algebraic to functional independence). Let $\mathbf{f} \subset \mathbb{F}_{q}[\mathbf{x}]$ be algebraically independent polynomials (wlog n-variate $n$ polynomials) with inseparable degree $p^{i}$. Then,

1. for all $t \geq p^{i}$, for random $\mathbf{a} \in \overline{\mathbb{F}}_{q}^{n}, f_{n}^{\leq t}(\mathbf{x}+\mathbf{a})$ cannot be written as $h \leq t\left(f_{1}(\mathbf{x}+\mathbf{a}), \ldots\right.$, $\left.f_{n-1}(\mathbf{x}+\mathbf{a})\right)$, for any $h \in \overline{\mathbb{F}}_{q}\left[Y_{1}, \ldots, Y_{n-1}\right]$.

2. for all $1 \leq t<p^{i}, \exists j \in[n]$, for random $\mathbf{a} \in \overline{\mathbb{F}}_{q}^{n}, f_{j}^{\leq t}(\mathbf{x}+\mathbf{a})$ can be written as $h_{j t}^{\leq t}\left(f_{1}(\mathbf{x}+\mathbf{a}), \ldots, f_{j-1}(\mathbf{x}+\mathbf{a}), f_{j+1}(\mathbf{x}+\mathbf{a}), \ldots, f_{n}(\mathbf{x}+\mathbf{a})\right)$, for some $h_{j t} \in \overline{\mathbb{F}}_{q}[\mathbf{Y}]$.

- Remark. Our proof works for any field $\mathbb{F}$ (manipulate in $\overline{\mathbb{F}}$ ). In case of characteristic $p \geq 2$ we get the above statement and in characteristic zero use inseparable degree $=1$.

Proof idea: By the hypothesis we have that each monomial $x_{j}^{p^{i}}, j \in[n]$, algebraically depends on $\mathbf{f}$ with a separable annihilating polynomial over $\mathbf{F}_{q}$. Consider ring $R:=\overline{\mathbb{F}}_{q}(\mathbf{z})[\mathbf{x}]$. The basic idea is to consider the minimal annihilating polynomial $A_{j}$ of $\left\{x_{j}^{p^{i}}, \mathbf{f}\right\}$ and formally shift the relevant polynomials by $\mathbf{z}$. From the proof of Thm.10 we get a functional dependence of $x_{j}^{p^{i}}$ on $\mathbf{f}(\mathbf{x}+\mathbf{z})$ up to any degree $t$.

Interestingly, when we take $t<p^{i}$ the monomial $x_{j}^{p^{i}}$ vanishes $\bmod \langle\mathbf{x}\rangle^{t+1}$. This means that the above yields, in fact, a functional dependence among $\mathbf{f}(\mathbf{x}+\mathbf{z})$.

On the other hand, for $t \geq p^{i}$, we get a nontrivial functional dependence of $x_{j}^{p^{i}}$ on $\mathbf{f}(\mathbf{x}+\mathbf{z})$, for all $j \in[n]$. In this case, one can give an argument using monomial ordering that there exists no functional dependence among $\mathbf{f}(\mathbf{x}+\mathbf{z})$.

We can see the classical Jacobian criterion as a special case of Theorems 10 and 13. The detailed discussions and missing proofs are given in the full version.

\section{Conclusion}

We give a criterion for testing algebraic independence over positive characteristic, in the spirit of Jacobian criterion, that works for any field. Its complexity is parameterized by the inseparable degree bound. It is also strong enough to give the inseparable degree at the same time. We give applications to locally low algebraic rank circuits in the cases that were open before.

\begin{tabular}{|c|c|c|}
\hline & Jacobian Criterion & Our Criterion \\
\hline The approach: & $\begin{array}{c}\text { reduces algebraic independence } \\
\text { to linear independence testing }\end{array}$ & $\begin{array}{c}\text { reduces algebraic independence } \\
\text { to linear independence testing }\end{array}$ \\
\hline Related "approximate" shift : & $\mathbf{f}(\mathbf{x}) \mapsto \mathbf{f}(\mathbf{x}+\mathbf{z}) \bmod \langle\mathbf{x}\rangle_{\mathbb{F}(\mathbf{z})[\mathbf{x}]}^{2}$ & $\mathbf{f}(\mathbf{x}) \mapsto \mathbf{f}(\mathbf{x}+\mathbf{z}) \bmod \mathcal{U}_{t}$ \\
\hline Vectors for $\mathbb{F}(\mathbf{z})$-dependence: & $\mathcal{H}_{1} \mathbf{f} \bmod \mathcal{U}_{1}$ & $\mathcal{H}_{t} \mathbf{f} \bmod \mathcal{U}_{t}$ \\
\hline Certifies alg.independence if: & $\mathbb{F}(\mathbf{x}) / \mathbb{F}(\mathbf{f})$ is separable & separable or inseparable $\mathbb{F}(\mathbf{x}) / \mathbb{F}(\mathbf{f})$ \\
\hline Efficiency in char $(\mathbb{F})=0:$ & randomized poly-time algorithm & $t=1$, same as Jacobian criterion $)$ \\
\hline $\begin{array}{c}\text { Efficiency in char }(\mathbb{F})=p, \\
\text { inseparable degree } \leq p^{e}:\end{array}$ & fails & $\begin{array}{c}\text { randomized poly }\left(\begin{array}{c}n+p^{e} \\
n\end{array}\right) \text {-time } \\
\text { algorithm }\end{array}$ \\
\hline
\end{tabular}


The main open problem is to investigate whether we can improve the criterion to get a randomized poly-time algorithm for circuits over a finite field. We mention a few special cases based on different restrictions on input. None of these cases are (efficiently) solved by presently known techniques.

- the polynomials are supersparse, i.e. sparse polynomials with possibly exponential degree.

- two bivariate circuits, with an exponentially large inseparable degree, over $\mathbb{F}_{2}$.

- $n$ quadratic polynomials over $\mathbb{F}_{2}$.

Our hitting-set result, for locally low algebraic rank circuits, still has a mild assumption on the characteristic. Can this be eliminated?

Acknowledgements. We thank Manindra Agrawal, Rohit Gurjar \& Arpita Korwar for the insightful discussions and encouragement. We thank Markus Bläser and the anonymous reviewers for the elaborate suggestions to improve the draft. NS acknowledges the support from DST/SJF/MSA-01/2013-14 and SB/FTP/ETA-177/2013.

\section{References}

1 M. Agrawal, C. Saha, R. Saptharishi, and N. Saxena. Jacobian hits circuits: Hitting-sets, lower bounds for depth-D occur-k formulas \& depth-3 transcendence degree-k circuits. In Proceedings of the 44th ACM Symposium on Theory of Computing (STOC), pages 599-614, 2012. (In SICOMP special issue).

2 Manindra Agrawal and V. Vinay. Arithmetic circuits: A chasm at depth four. In 49th Annual IEEE Symposium on Foundations of Computer Science, FOCS, pages 67-75, 2008.

3 W. Bauer and V. Strassen. The complexity of partial derivatives. Theoretical Computer Science, 22(3):317-330, 1983.

4 M. Beecken, J. Mittmann, and N. Saxena. Algebraic Independence and Blackbox Identity Testing. Inf. Comput., 222:2-19, 2013. (Conference version in ICALP 2011).

5 Radu Curticapean. Counting matchings of size k is \#W[1]-hard. In Automata, Languages, and Programming, pages 352-363. Springer, 2013.

6 Richard A DeMillo and Richard J Lipton. A probabilistic remark on algebraic program testing. Information Processing Letters, 7(4):193-195, 1978.

7 Z. Dvir, A. Gabizon, and A. Wigderson. Extractors and rank extractors for polynomial sources. Comput. Complex., 18(1):1-58, 2009. (Conference version in FOCS 2007).

8 Z. Dvir, D. Gutfreund, G.N. Rothblum, and S.P. Vadhan. On approximating the entropy of polynomial mappings. In Innovations in Computer Science (ICS), pages 460-475, 2011.

9 Zeev Dvir. Extractors for varieties. In Proceedings of the 24th IEEE Conference on Computational Complexity (CCC), pages 102-113, 2009.

10 Zeev Dvir, Swastik Kopparty, Shubhangi Saraf, and Madhu Sudan. Extensions to the method of multiplicities, with applications to kakeya sets and mergers. SIAM Journal on Computing, 42(6):2305-2328, 2013. (Preliminary version in FOCS'09).

11 Zeev Dvir, Amir Shpilka, and Amir Yehudayoff. Hardness-randomness tradeoffs for bounded depth arithmetic circuits. SIAM Journal on Computing, 39(4):1279-1293, 2009.

12 Richard Ehrenborg and Gian-Carlo Rota. Apolarity and canonical forms for homogeneous polynomials. European Journal of Combinatorics, 14(3):157-181, 1993.

13 Michael A Forbes. Polynomial identity testing of read-once oblivious algebraic branching programs. PhD thesis, Massachusetts Institute of Technology, 2014.

14 Michael A Forbes. Deterministic divisibility testing via shifted partial derivatives. In Foundations of Computer Science (FOCS), 2015 IEEE 56th Annual Symposium on, pages 451-465. IEEE, 2015. 
15 Krister Forsman. Two themes in commutative algebra: Algebraic dependence and kähler differentials, 1992.

16 Ankit Gupta, Pritish Kamath, Neeraj Kayal, and Ramprasad Saptharishi. Arithmetic circuits: A chasm at depth three. In 54th Annual IEEE Symposium on Foundations of Computer Science, FOCS 2013, 26-29 October, 2013, Berkeley, CA, USA, pages 578-587, 2013.

17 Helmut Hasse and Friedrich K. Schmidt. Noch eine begründung der theorie der höheren differentialquotienten in einem algebraischen funktionenkörper einer unbestimmten. (nach einer brieflichen mitteilung von f.k.schmidt in jena). Journal für die reine und angewandte Mathematik, 177:215-223, 1937.

18 L. Illusie. Crystalline cohomology. In Proc. Sympos. Pure Math., volume 55, pages 43-70, 1994. Motives (Seattle, WA, 1991).

19 I Martin Isaacs. Algebra: a graduate course, volume 100. American Mathematical Soc., 1994.

20 C. G. J. Jacobi. De determinantibus functionalibus. J. Reine Angew. Math., 22(4):319-359, 1841.

21 K. A. Kalorkoti. A Lower Bound for the Formula Size of Rational Functions. SIAM J. Comp., 14(3):678-687, 1985. (Conference version in ICALP 1982).

22 N. Kayal. The Complexity of the Annihilating Polynomial. In Proceedings of the 24th Annual IEEE Conference on Computational Complexity (CCC), pages 184-193, 2009.

23 Neeraj Kayal, Nutan Limaye, Chandan Saha, and Srikanth Srinivasan. An exponential lower bound for homogeneous depth four arithmetic formulas. In Foundations of Computer Science (FOCS), IEEE 55th Annual Symposium on, pages 61-70. IEEE, 2014.

24 Anthony W Knapp. Advanced algebra. Springer Science \& Business Media, 2007.

25 Steven G Krantz and Harold R Parks. The implicit function theorem: history, theory, and applications. Springer Science \& Business Media, 2012.

26 Mrinal Kumar and Shubhangi Saraf. On the power of homogeneous depth 4 arithmetic circuits. In Foundations of Computer Science (FOCS), IEEE 55th Annual Symposium on, pages 364-373. IEEE, 2014.

27 Mrinal Kumar and Shubhangi Saraf. Arithmetic circuits with locally low algebraic rank. Electronic Colloquium on Computational Complexity (ECCC), 22:194, 2015. (To appear in CCC 2016). URL: http://eccc.hpi-web.de/report/2015/194.

28 M.S. L'vov. Calculation of invariants of programs interpreted over an integrality domain. Cybernetics and Systems Analysis, 20:492-499, 1984.

29 Johannes Mittmann, Nitin Saxena, and Peter Scheiblechner. Algebraic independence in positive characteristic: A p-adic calculus. Transactions of the American Mathematical Society, 366(7):3425-3450, 2014.

30 James G Oxley. Matroid theory, volume 3. Oxford university press, 2006.

31 O. Perron. Algebra I (Die Grundlagen). W. de Gruyter, Berlin, 1927.

32 A. Płoski. Algebraic Dependence of Polynomials After O. Perron and Some Applications. In Computational Commutative and Non-Commutative Algebraic Geometry, pages 167-173. 2005.

33 Ran Raz. Elusive functions and lower bounds for arithmetic circuits. In Proceedings of the fortieth annual ACM symposium on Theory of computing, pages 711-720. ACM, 2008.

34 Ramprasad Saptharishi. A survey of lower bounds in arithmetic circuit complexity, 2016. https://github.com/dasarpmar/lowerbounds-survey/.

35 Nitin Saxena. Progress on polynomial identity testing-ii. In Perspectives in Computational Complexity, pages 131-146. Springer, 2014.

36 J.T. Schwartz. Fast probabilistic algorithms for verification of polynomial identities. J. $A C M, 27(4): 701-717,1980$. 
37 A. Shpilka and A. Yehudayoff. Arithmetic Circuits: A survey of recent results and open questions. Foundations and Trends in Theoretical Computer Science, 5(3-4):207-388, 2010.

38 Oswald Teichmüller. Differentialrechnung bei charakteristik p. Journal für die reine und angewandte Mathematik, 175:89-99, 1936.

39 William Nathaniel Traves. Differential operators and Nakai's conjecture, 1998.

40 Richard Zippel. Probabilistic algorithms for sparse polynomials. Springer, 1979. 